http://dx.doi.org/10.4314/ajtcam.v10i4.30

\title{
SPECIFIC NEUROPROTECTIVE EFFECTS OF MANUAL STIMULATION OF REAL ACUPOINTS VERSUS NON-ACUPOINTS IN RATS AFTER MIDDLE CEREBRAL ARTERY OCCLUSION
}

\author{
Xin Ling Li ${ }^{1,2}$, Nong Xiao Fan ${ }^{3}$, Hong Zhi Meng ${ }^{3}$, Min Xue Shi ${ }^{3,}$, Ding Luo ${ }^{1}$, \\ Nan Ya Zhang ${ }^{1}$
}

\author{
${ }^{1}$ Postgraduate Department of Tianjin University of Traditional Chinese Medicine, Tianjin 300193, \\ China, ${ }^{2}$ Department of Rehabilitation Medicine, West China Medical School, West China Hospital \\ Sichuan University, 37 Guoxue Xiang, Wuhou District, Chengdu 610041, Sichuan, China., \\ ${ }^{3}$ Department of Acupuncture and Moxibustion, First Hospital Affiliated to Tianjin University of \\ Traditional Chinese Medicine, Tianjin 300193, China. \\ *E-mail: tjdrshi@msn.com
}

\begin{abstract}
The objective of this study was to investigate the effectiveness and specific effects of acupuncture on ischemic-induced damage in rats after permanent middle cerebral artery occlusion. Cerebral ischemia was induced by middle cerebral artery occlusion in male Wistar rats. The rats were divided into the following 4 groups: normal controls, ischemic, real acupuncture-treated (Shuigou, DU26), and non-acupoint-treated groups. On the third postoperative day, neurological deficit scores, cerebral blood flow, infarction volume, and neuronal cell death counts were measured. In the real acupuncture-treated group, the neurological deficit scores and cerebral blood flow were improved $(\mathrm{p}<0.05)$ and the infarction volume and neuronal cell death counts were reduced ( $\mathrm{p}<0.01$ ) compared to the ischemic and non-acupoint-treated groups. The present study demonstrated that real acupuncture was effective against focal ischemia-induced damage in rats after middle cerebral artery occlusion, and the effects were specifically related to the right needling location.
\end{abstract}

Key words: specificity, real acupoint, non-acupoint, middle cerebral artery occlusion, animal experimentation

\begin{abstract}
Abbreviations: CBF, cerebral blood flow; HE, hematoxylin and eosin; LDF, laser Doppler flowmetry; MCAO, middle cerebral artery occlusion; NDS, neurological deficit score; N-ACU, non-acupoint; NORM, normal; R-ACU, real acupuncture; TCM, traditional Chinese medicine; TTC, 2,3,5-triphenyltetrazolium chloride
\end{abstract}

\section{Introduction}

Stroke is the third leading cause of death and the leading cause of severe disability in Western high-income countries, even with the declining trend in the incidence rates over the past 4 decades (Feigin et al., 2009). In China, the age-standardised incidence rate of stroke increased by 6.7\% annually from 1984 to 2004 (Zhao et al., 2008). Furthermore, it remains the second or third leading cause of death in China, and it has resulted in long-term disability in recent years (He et al., 2005).

Acupuncture, which has been used for about 3,000 years in China, has become a global therapeutic method for many clinical conditions, especially stroke, in recent decades. It has been reported that acupuncture has neuroprotective effects, such as improving post-stroke perception, enhancing independence, and promoting the recovery of neurological function in patients with stroke (Hopwood et al., 2005; Si et al., 1998).

Current evidence has shown that its use is safe and feasible during stroke recovery (Rao et al., 2006). Several 
http://dx.doi.org/10.4314/ajtcam.v10i4.30

mechanisms that underlie these benefits have been suggested in clinical and animal experiments. For example, it has been reported that acupuncture treatment can increase activity in the ipsilesional motor cortex (Schaechter et al., 2007), increase cerebral thioredoxin expression (Siu et al., 2005), promote the activities of respiratory enzymes and reduce the generation of reactive oxygen species, resulting in improvement of respiratory chain function and anti-oxidative capability of brain tissues in the infarct penumbra zone (Zhong et al., 2009). For other clinical conditions, a systematic review concluded that acupuncture should be advocated as an adjunct to conventional care for the treatment of chronic low back pain (Yuan et al., 2008). Although many studies have reported that the single or combinative administration of acupuncture provides significant therapeutic effects in many clinical conditions, the effectiveness of acupuncture as a treatment intervention remains largely untested and unproved. The effectiveness and effect specificity of acupuncture is still an equivocal and controversial issue.

Some systematic reviews have suggested that the current evidence is not sufficiently extensive or rigorous to support the routine use of acupuncture for the treatment of acute stroke or during stroke rehabilitation (Kong et al., 2010; Wu et al., 2010). One meta-analysis has shown that acupuncture has only a small positive effect on disability and no effect on motor recovery (Sze et al., 2002). One study has reported that real acupuncture is not superior to sham acupuncture (without penetrating the skin) for stroke recovery (Park et al., 2005). In consideration of these findings, the present study was designed to examine rats that were not treated with acupuncture and rats that were treated with invasive non-acupoint acupuncture in a nonchannel and nonpoint location. These rats were used as controls and were compared to rats that were treated with acupuncture. This study was designed to identify the effectiveness of acupuncture and to determine if the specific effects of acupuncture are based on needling in an accurate location as defined by Traditional Chinese Medicine (TCM) theories.

According to TCM theory, the acupuncture point of Shuigou (DU26) has prominent therapeutic effects in brain disorders. Animal models of middle cerebral artery occlusion (MCAO) are widely used in the exploration of the pathophysiology of ischemic brain injury and the evaluation of the beneficial effects of intervention methods. Hence, the present study selected the DU26 acupoint as the point of real acupuncture in the treatment of MCAO rats and used the site that was $5 \mathrm{~mm}$ to the left of the DU26 location as the non-acupoint acupuncture control in order to explore the specific effects of real acupuncture treatment.

The purpose of the present study was to evaluate the neurological deficit score (NDS), cerebral blood flow (CBF), infarct volume, and the histological changes in permanent MCAO rats after a 3-day acupuncture treatment and thereby to investigate the efficacy and the effects specificity of manual stimulation to acupoint DU26 versus a non-acupoint.

\section{Materials and Methods}

\section{Animals}

The experiments were conducted on adult male Wistar rats that were purchased from the WeiTong-LiHua Experimental Animal Technical Limited Corporation (Beijing, China) in September 2007 and that weighed 250-280 g at the time of the surgical operation. The rats were housed in the animal centre of the First Hospital Affiliated with Tianjin University of TCM with free access to food and water, and they were maintained on a 12-h light/dark cycle. The humidity level was $55 \pm 5 \%$, and the indoor temperature was maintained at $22 \pm 1^{\circ} \mathrm{C}$. All of the protocols of the present study were performed in accordance with the guidelines of the Laboratory Animal Ethics Committee that was approved by Tianjin University of TCM. All efforts were made to minimise animal suffering at each stage of the current experiment and to reduce the number of animals that were used. The whole study was performed on 72 animals. This number did not take into account the mortality of animals resulting from surgery. All of the animals were randomly assigned to 4 groups: normal (NORM), ischemic (MCAO), non-acupoint (N-ACU), and real acupuncture (DU26) (R-ACU), with $\mathrm{n}=18$ per group. At the end of treatment on the third day, NDS and CBF tests were conducted on all animals. In each group, tissues from 12 rats were stained with 2,3,5-triphenyltetrazolium chloride (TTC), and tissues from 6 other animals were stained with hematoxylin and eosin (HE). 


\section{MCAO Operation}

The MCAO operation was conducted with the improved intraluminal suture method that was originally described by Longa et al. (1989). Briefly, rats were anesthetised with 10\% chloral hydrate (1 mL/200 g, i.p.) and placed in dorsal recumbence. A 20- to 25-mm-long longitudinal incision was made in the neck to expose the left carotid bifurcation. The left external carotid artery and the left internal carotid artery were carefully dissected from the surrounding tissues. A 4.0 nylon monofilament (40 mm in length and $0.265 \mathrm{~mm}$ in diameter) that was coated with silicone was inserted from the external carotid artery into the left internal carotid artery in order to block the origin of the left middle cerebral artery. Animal body temperature was maintained at $37 \pm 1^{\circ} \mathrm{C}$ during and after the operation. All animals received injections of a few drops of gentamycin sulphate on the incision and $1 \mathrm{~mL}$ of physiological saline subcutaneously shortly after the surgery in order to prevent postoperative infections or dehydration. The animals recovered rapidly from the anesthesia and were returned to their home cages. The rats were decapitated $72 \mathrm{~h}$ after the operation.

\section{Acupuncture Stimulation}

Acupuncture stimulation, which consisted of dry needle acupuncture, was performed by 2 skilled Acupuncture and Moxibustion major postgraduates who had trained for 5 - 7 years in theoretical and clinical settings. The acupuncture was started immediately after the baseline NDS assessment. A stainless-steel needle, which was $0.25 \mathrm{~mm}$ in diameter and $40 \mathrm{~mm}$ in length (Suzhou Medical Supplies Factory Co., Ltd., Jiangsu, China), was manually inserted 5 mm deep into DU26 or the non-acupoint. The needle was thrust and lifted at a frequency of $3 \mathrm{~Hz}$ (i.e., 180 per min) for $5 \mathrm{~s}$, as described in a previous study (Li Ling-xin et al., 2010). The acupuncture treatment was applied at DU26 or the non-acupoint twice a day for 3 days. The location of DU26 and the non-acupoint is shown in Figure 1.

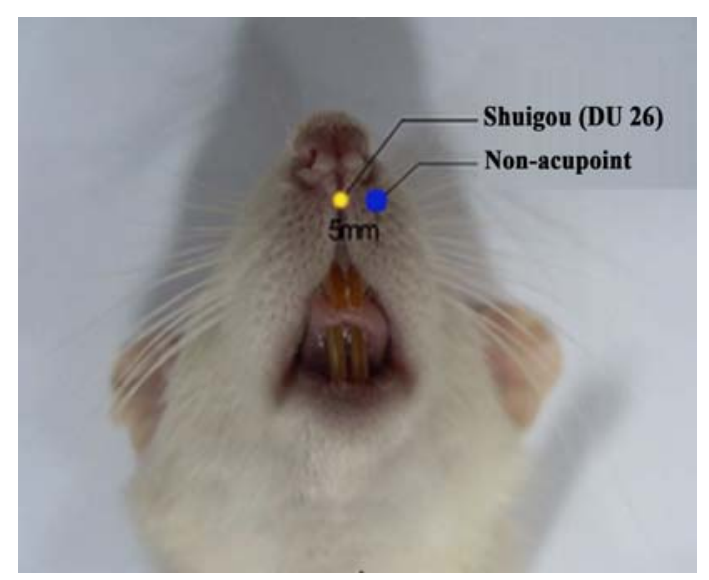

Figure 1: Location of the DU26 acupoint and the non-acupoint. DU26 is indicated by the yellow dot that is located at the junction of the upper one-third and lower two-thirds of the cleft lip midline. The non-acupoint, which is indicated as a blue dot, was chosen at a site that was $5 \mathrm{~mm}$ to the left of DU26.

\section{NDS Evaluation}

NDS was assessed by a researcher who was not involved in the animal treatments and who was blind to the experimental group before and after acupuncture treatment. The neurological function of all animals was evaluated with the following: Zausinger 6-point scale (Zausinger et al., 2000): 0 points, no spontaneous activity; 1 point, spontaneous circling; 2 points, circling if pulled by tail; 3 points, lowered resistance to lateral push; 4 points, contralateral forelimb flexion; and 5 points, no deficits.

\section{CBF Measurement}

In the present study, regional CBF was monitored in the ischemic cortex with laser Doppler flowmetry (LDF). The LDF technique has been used in many studies (Lou et al., 2004; Yang et al., 1994). In brief, laser light from a semiconductor laser 
http://dx.doi.org/10.4314/ajtcam.v10i4.30

diode (wavelength, $785 \mathrm{~nm}$; maximal energy acquired, $2.5 \mathrm{~mW}$ ) in a flexible fibre optic is used to detect moving red blood cells. In this experiment, the regional CBF was measured with a DRT4 Laser Doppler Perfusion and Temperature Monitor (made in Moor Instruments, Devon, UK and purchased from Gene \& I Life Science Co., Ltd., Beijing, China). The left dorsolateral portion of the calvaria was carefully abraded with a mini-drill until only a paper-thin layer of bone remained in order to prevent cerebral spinal fluid leakage. A hole that was $2.0 \mathrm{~mm}$ in diameter at $3.0 \mathrm{~mm}$ lateral and $1.0 \mathrm{~mm}$ caudal to the bregma was then drilled. Then, each animal was placed in a supine position, and the head was firmly immobilised in a stereotaxic frame. A Laser-Doppler probe, which was held in a micromanipulator, gently touched the intact dura mater in order to measure the regional CBF. The flow values were recorded during 1-min periods every 10 min until the LDF baseline was stable, and the data were calculated as averaged values.

\section{Morphometric Measurements of Infarct Volume}

The area of the cerebral infarction was quantified by TTC staining. Twelve rats in each group were euthanized immediately after the 3-day treatment and decapitated. The brain was swiftly extracted and refrigerated at $-20^{\circ} \mathrm{C}$ for $30 \mathrm{~min}$. The brain that was detached from the olfactory bulb, cerebellum, and lower brain stem was sectioned into 2-mm-thick slices starting at the frontal pole with a Brain Matrix Slicer (The Vibratome Company, St Louis, MO, USA). Slices were immersed in a 2\% TTC (Tianjin RuiJingTe Chemicals Co., Ltd., Tianjin, China) solution in a Petri dish and incubated at $37^{\circ} \mathrm{C}$ for $20 \mathrm{~min}$ in order to optimise TTC staining. The sections were then placed in 12\% formalin for fixation for 2 min. Finally, the TTC-stained slices were photographed with an Olympus fe-240 digital camera (Shanghai Pooher Photoelectric Technology Co., Ltd., Shanghai, China) and analysed by Image Analysis Software (Image-pro Plus 6.0, Media Cybernetics, Inc., Bethesda, MD, USA). Infarction volume was determined as a percentage of the total ipsilateral hemispheric volume, as described by Swanson et al. (1990) and O’Donnell et al. (2004). The lesion volume was calculated as a percentage of the lesion volume compared to the contralateral hemisphere volume. Briefly, the volume of the lesion was calculated with the following formula:

$$
\left[\left(\mathrm{V}_{\mathrm{C}}-\mathrm{V}_{\mathrm{L}}\right) / \mathrm{V}_{\mathrm{C}}\right] \times 100 \%,
$$

where $\mathrm{V}_{\mathrm{C}}$ is the volume of the noninfarcted tissue in the contralateral hemisphere and $\mathrm{V}_{\mathrm{L}}$ is the volume of the lesioned tissue in the infarcted ipsilateral hemisphere. The analysis was conducted under blinded conditions.

\section{HE Histological Analysis}

The remaining 6 rats in each group were anesthetised with 10\% chloral hydrate intraperitoneally as described above and then decapitated. The brains were quickly removed from the skulls, fixed in $12 \%$ neutral formalin for $24 \mathrm{~h}$, and then cut into 3-mm-thick coronal slices for routine histopathological examinations of HE staining by light microscopy at a magnification of 400×. Briefly, two 3-mm-thick coronal brain sections from the anterior and posterior $3 \mathrm{~mm}$ from the midpoint of the cerebral longitudinal fissure of each brain were placed in different concentrations of ethanol and xylol for dehydration and then embedded in paraffin in order to make paraffin blocks. Sections from the paraffin blocks were cut at a thickness of $5 \mu \mathrm{m}$ and stained with HE. Neurons were examined under a light microscope at a magnification of $400 \times$ by a person who was blinded to the study. The numbers of dead neurons, which were counted on 2 histological sections from each animal, were expressed as the average number of dead neuronal cells.

\section{Statistical Analysis}

The data were analysed with the Statistical Package for the Social Sciences (IBM Corporation, Armonk, NY, USA). The NDS data are presented as a median (interquartile range). For the comparisons between the groups, the data were analysed with a K independent samples nonparametric test (Kruskal-Wallis Test), which was followed by a post-hoc Mann-Whitney U-test. For the comparisons with the baseline, the NDS data were analysed with the 2-related-samples nonparametric test (Wilcoxon Test). The other data are presented as mean \pm standard deviation, and the differences between the groups were 
assessed with one-way analyses of variance followed by the Least Significant Difference test if there was homogeneity of variance or the Tamhane's T2 test if there was heterogeneity in the variance for multiple comparisons. The criterion for statistical differences was $\mathrm{p}<0.05$.

\section{Experimental Flowchart}

The whole experiment was completed in a week. All the animals were fed for 3 days. When the MCAO operations were completed, all animals were randomly assigned to the NORM, the MCAO, the N-ACU, or the R-ACU groups, and the NORM animals were only handled outside of their cages during the time of the operation. At that time, the first neurological function evaluation was assessed. Then, the N-ACU and R-ACU animals were treated with sham or real acupuncture, respectively, twice a day for 3 days. On the third day and after treatment was completed, the NDS and CBF examinations were performed in all animals. Then, in each group, 12 rats were decapitated for TTC staining, and the other 6 rats were decapitated for HE staining. A flowchart of the procedures in this experiment is displayed in Figure 2.

\section{Experimental Flowchart}

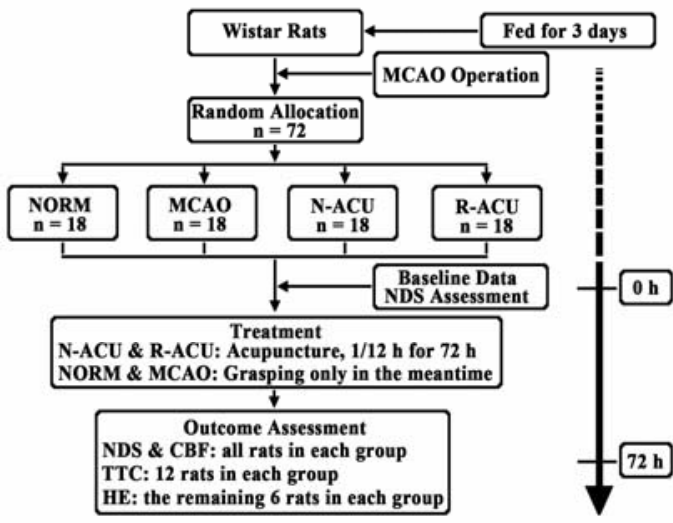

Figure 2: Experimental Flowchart. MCAO, middle cerebral artery occlusion; NORM, normal (no MCAO);

N-ACU, MCAO + stimulation to the non-acupoint;

R-ACU, MCAO + stimulation to DU26;

NDS, neurological deficit score;

CBF, cerebral blood flow;

TTC, 2,3,5-triphenyltetrazolium chloride;

HE, hematoxylin \& eosin

\section{Results}

\section{NDS}

Behavioural tests are used to reflect the neurological deficits in rat brain function. NDS was expressed as a median (interquartile range) in this experiment. Compared to baseline, we observed that NDS was significantly increased after real acupuncture treatment, while there were no obvious differences in the other 3 groups. Furthermore, when comparing the groups after treatment, NDS in the R-ACU group was significantly increased [3(1)] compared to those in the MCAO [2(1)] and the N-ACU [2(1)] groups ( $<<0.05$ for both). Slight differences were observed between the MCAO and N-ACU groups, but these differences were not significant ( $p>0.05)$. The NDS data are shown in Figure 3.

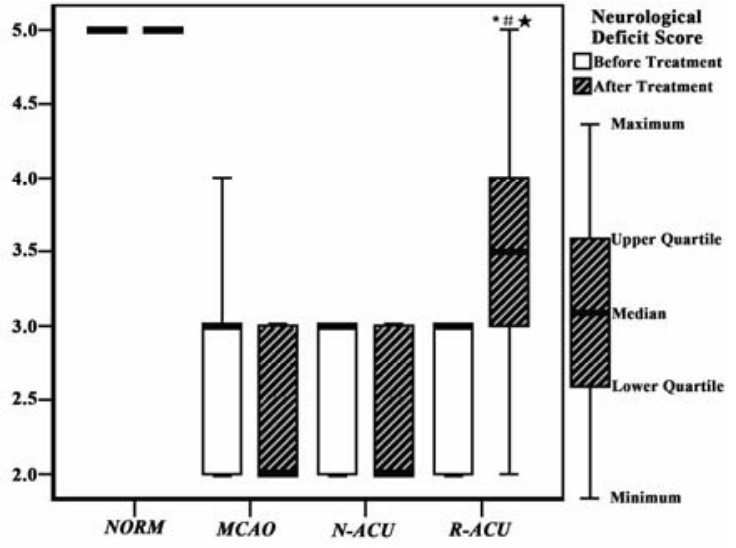

Figure 3: Results of the NDS assessments in each group and explanation of the box plot.

Neurological examinations were conducted before and after treatment, and the values are presented as box plots. The groups were as follows: NORM ( $\mathrm{n}=$ 18); MCAO ( $=18) ; \mathrm{N}-\mathrm{ACU}(\mathrm{n}=18) ; \mathrm{R}-\mathrm{ACU}(\mathrm{n}=$ 18). ${ }^{*} \mathrm{p}<0.05$ versus MCAO; \#p $<0.05$ versus $\mathrm{N}-\mathrm{ACU} ;{ }^{\star} \mathrm{p}<0.05$ versus baseline. See abbreviation explanation in Figure 2. 
CBF

Occlusion of the left middle cerebral artery led to an immediate sharp drop in the regional CBF in the ischemic cortex (219.94 \pm 13.65$)$ in MCAO rats compared to that (417.59 \pm 17.28$)$ in NORM rats. A tendency to return to normal levels of CBF was observed in both R-ACU and N-ACU rats after acupuncture intervention (233.81 \pm 15.76 in N-ACU rats and 299.16 \pm 25.31 in R-ACU rats). However, the CBF values in R-ACU rats were significantly recovered compared to that in N-ACU rats $(\mathrm{p}<0.05)$. The difference in CBF between the R-ACU and MCAO groups was significant $(\mathrm{p}<0.05)$. In contrast, no significant difference in regional CBF was seen between the N-ACU and MCAO groups ( $>0.05)$. The CBF data are shown in Figure 4.

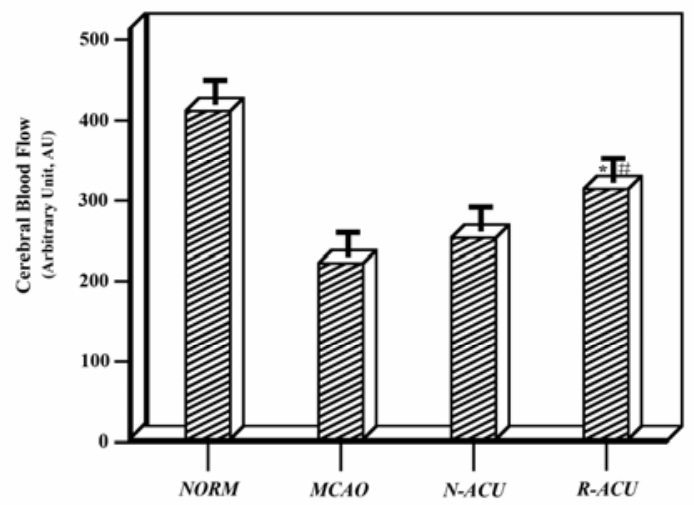

Figure 4: Bar graph showing the regional $\mathrm{CBF}$ changes in ischemic cortex after the 3-day treatment in the 4 groups.

The values are expressed as mean \pm standard deviation. In each group, $\mathrm{n}=18 .{ }^{*} \mathrm{p}<0.05$ versus MCAO; \#p $<0.05$ versus N-ACU. See abbreviation explanation in Figure 2.

\section{Infarction Volume}

In the evaluation of infarct volume, TTC staining that was conducted $72 \mathrm{~h}$ after ischemia demonstrated the pathological features of ischemic damage that is induced by MCAO. Figure 5A shows representative photomicrographs of coronal sections that were obtained from rats from the 4 groups. No cerebral infarctions were observed in the NORM group. The white areas in the figure indicate the typical infarcted area in the other 3 groups. Figure 5B shows that the NORM rats did not show any evidence of brain lesioning $(0.00 \pm 0.00 \%)$. However, in the MCAO group, severe cerebral infarctions were observed in all rats, and the infarct ratio was $22.67 \pm 2.15 \%$, which was significantly different compared to the NORM group $(\mathrm{p}<0.05)$. Although the N-ACU $(19.48 \pm 1.74 \%)$ and the R-ACU $(8.93 \pm 1.02 \%)$ rats showed a decrease in brain infarct volume compared to the MCAO group, the R-ACU rats were significantly different $(\mathrm{p}<0.05)$ compared to both the MCAO and $\mathrm{N}$-ACU groups, while the difference in the brain infarct area between the N-ACU and MCAO groups was not statistically significant $(\mathrm{p}>0.05)$. 

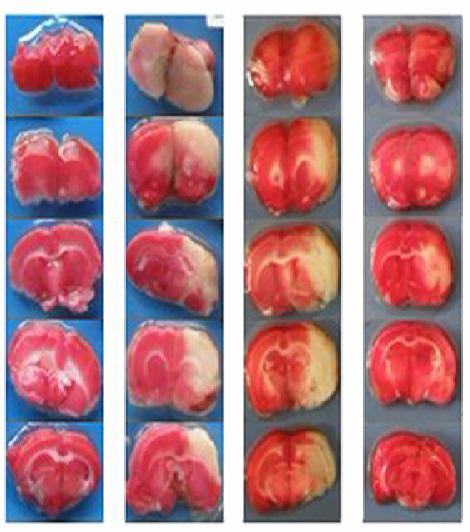

NORM MCAO N-ACU R-ACU

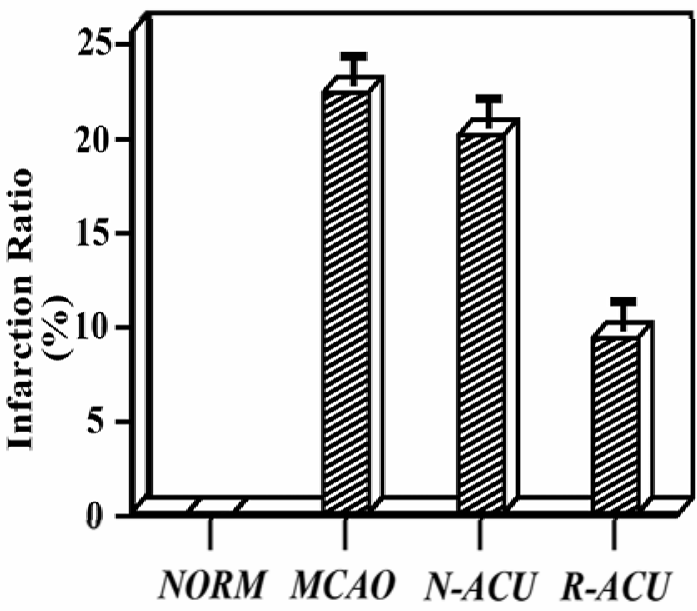

B

Figure 5: The effects of acupuncture on infarction volume induced by MCAO in rats from each of the groups. A: Representative photographs of coronal sections obtained from the rats in the 4 groups. The sections displayed illustrate the extent of the typical infarction tissue and the approximate mean infarct volume in each group. The white areas indicate the infarcted brain tissue. B: Bar graph of the infarction volume. The data are expressed as mean \pm standard deviation $(\mathrm{n}=18$ per group). *p $<0.05$ versus MCAO; \#p $<0.05$ versus N-ACU. See abbreviation explanation in Figure 2.

\section{Neuronal Cell Death Count}

In the light microscopic histological evaluation, the necrotic areas in R-ACU rats were not widespread, and the number of dead neurons in R-ACU rats (5 \pm 0.38) was significantly less than those in MCAO (20 \pm 0.56$)$ and N-ACU $(19 \pm 0.67, \mathrm{p}<$ 0.05 for both) rats. Compared to the NORM rats ( $2 \pm 0.42)$, the number of dead neurons was significantly different in the MCAO rats and in the N-ACU rats ( $\mathrm{p}<0.05$ for both). The difference in the dead neuron count between the N-ACU and MCAO groups was not statistically significant $(\mathrm{p}>0.05)$. The light microscopic results are shown in Figure 6.

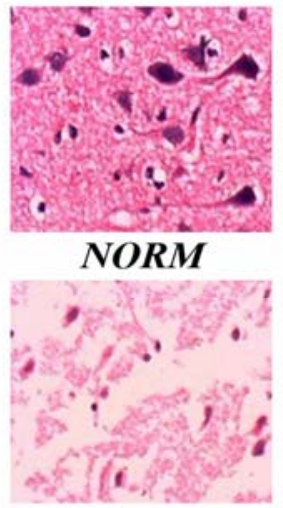

$N-A C U$

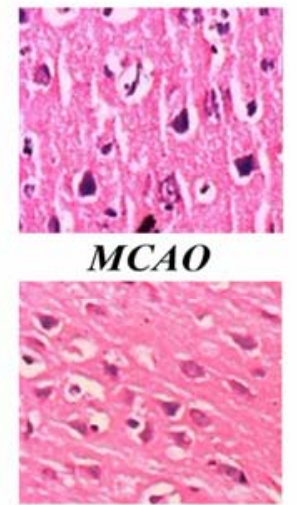

$R-A C U$ A

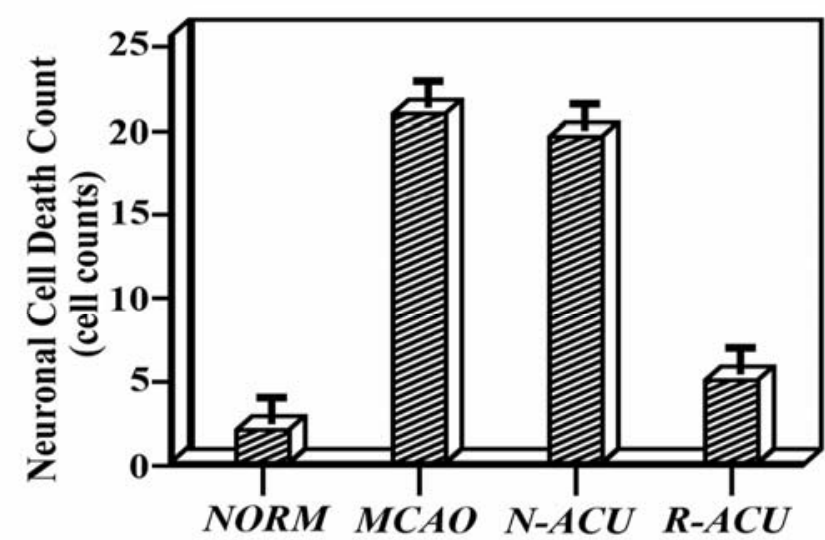

B

Figure 6: The effects of acupuncture on the count of dead neurons in the 4 groups 3 days after MCAO

A: Representative HE-stained sections from 1 rat in each group

B: Bar graph showing the neuronal count of dead neurons. The data is expressed as means \pm standard deviation ( $\mathrm{n}=18$ per group). ${ }^{*} \mathrm{p}<0.05$ versus MCAO; $\# \mathrm{p}<0.05$ versus $\mathrm{N}$-ACU. See abbreviation explanation in Figure 2 


\section{Discussion}

Acupuncture has been used for thousands of years in China, and its use has been increasing in other countries and territories worldwide in recent years. Nevertheless, there have been many lines of evidence that have shown that any definite conclusions about the effectiveness of acupuncture and the specificity of acupuncture treatment effects are equivocal and controversial. Two stroke trials on the efficacy of acupuncture did not provide enough proof to support the theory that acupuncture has a beneficial effect on acute stroke patients or for stroke rehabilitation (Gosman-Hedström et al., 1998; Johansson et al., 2001). In other clinical conditions, 3 randomised control trials reported statistically non-significant results and failed to find benefits of acupuncture (Painovich et al., 2012; Thomas et al., 2006; Vickers et al., 2005). There has also been a lack of powerful evidence of the specificity of acupuncture effects. The main outcome of the German Acupuncture Research Programs, which consisted of 4 trials examining patients with low back pain (Haake et al., 2007), gonarthrosis, migraines (Diener et al., 2006), and tension-type headaches and which was performed between 2001 and 2006, suggested that the differences between real and sham acupuncture were not significant in any trial. In addition, the Stop Hypertension with the Acupuncture Research Program (Macklin et al., 2006), which enrolled 192 participants with mild to moderate hypertension and which was conducted in the US, obtained results that the primary outcome (mean blood pressure) did not differ significantly between real and sham acupuncture treatment groups. The conclusions of the above studies suggest that acupuncture effects are not definite and are non-specific. In order to investigate the hypothesis that acupuncture is effective and its effects are specifically related to the right needling location, the present study was designed to compare real acupuncture with invasive non-acupoint acupuncture and no treatment in MCAO rats.

In our study, we found that, compared to baseline, NDS was significantly increased after real acupuncture treatment, while there were no obvious differences among the other 3 groups. Thus, a significant improvement on neurological functional outcome was observed in animals treated with real acupuncture twice a day for 3 days after MCAO. Animals with no treatment and non-acupoint acupuncture treatment exhibited similar neurological deficits, which were significantly decreased in both of these groups compared to the normal rats. When we measured the CBF in the ischemic zone with LDF at the end of the intervention, the average CBF in the ischemic lateral cortex of MCAO and N-ACU rats had transparently dropped compared to the NORM rats, while the CBF of the R-ACU rats was significantly increased compared to the MCAO and N-ACU rats. Observations of the morphological alterations in the rats' brains showed that significant histopathological improvements were found in the R-ACU rats. For the percentage of infarction ratio, real acupuncture treatment resulted in a significant decrease compared to the N-ACU rats, while the neuronal cell death counts in both the striatum and the motor cortex were also obviously decreased in rats treated with real acupuncture. Thus, all of the data from the current study showed that real acupuncture treatment was more effective than non-acupoint acupuncture in improving the NDS, increasing CBF and protecting neuronal cells against the ischemic-induced damage seen in MCAO rats. Furthermore, the neurological function improvements might have been associated with increased CBF or decreased infarction volume. These findings verify the trial's hypothesis that there are differences in the specific physiological effects between the real acupoint and the non-acupoint. However, the evidence from the present study did not explain the mechanisms of the acupuncture effects on molecular signal transduction and gene expression levels. Therefore, evidence of the molecular and genetic mechanisms of acupuncture should be the aim of research in the future.

In conclusion, our present results showed that real acupuncture improved the NDS, increased the focal CBF, and markedly reduced the infarction volume in MCAO rats. These findings suggested that real acupuncture is effective in providing neuroprotective effects against ischemic-induced damage in rats after MCAO and that its effects were specifically related to the right needling location. 
http://dx.doi.org/10.4314/ajtcam.v10i4.30

\section{Acknowledgement}

The work was funded by China’s National Key Basic Research Program (973 Program, No. 2006CB504504; No. 2010CB530506).

\section{References}

1. Diener, H. C., Kronfeld, K., Boewing, G., Lungenhausen, M., Maier, C., Molsberger, A., Tegenthoff, M., Trampisch, H. J., Zenz, M. and Meinert, R. (2006). Efficacy of acupuncture for the prophylaxis of migraine: a multicentre randomised controlled clinical trial. Lancet Neurol., 5: 310 - 316.

2. Feigin, V. L., Lawes, C. M., Bennett, D. A., Barker-Collo, S. L. and Parag, V. (2009). Worldwide stroke incidence and early case fatality reported in 56 population-based studies: a systematic review. Lancet Neurol., 8: 355 - 369.

3. Gosman-Hedstrom, G., Claesson, L., Klingenstierna, U., Carlsson, J., Olausson, B., Frizell, M., Fagerberg, B. and Blomstrand, C. (1998). Effects of acupuncture treatment on daily life activities and quality of life: a controlled, prospective, and randomized study of acute stroke patients. Stroke, 29: 2100 - 2108.

4. Haake, M., Muller, H. H., Schade-Brittinger, C., Basler, H. D., Schafer, H., Maier, C., Endres, H. G., Trampisch, H. J. and Molsberger, A. (2007). German Acupuncture Trials (GERAC) for chronic low back pain: randomized, multicenter, blinded, parallel-group trial with 3 groups. Arch Intern Med., 167: 1892 - 1898.

5. He, J., Gu, D., Wu, X., Reynolds, K., Duan, X., Yao, C., Wang, J., Chen, C. S., Chen, J., Wildman, R. P., Klag, M. J. and Whelton, P. K. (2005). Major causes of death among men and women in China. N Engl J Med., 353: 1124 - 1134.

6. Hopwood, V. and Lewith, G. T. (2005). Does acupuncture help stroke patients become more independent? J Altern Complement Med., 11: 175 - 177.

7. Johansson, B. B., Haker, E., von Arbin, M., Britton, M., Langstrom, G., Terent, A., Ursing, D. and Asplund, K. (2001). Acupuncture and transcutaneous nerve stimulation in stroke rehabilitation: a randomized, controlled trial. Stroke, 32: 707 - 713.

8. Kong, J. C., Lee, M. S., Shin, B. C., Song, Y. S. and Ernst, E. (2010). Acupuncture for functional recovery after stroke: a systematic review of sham-controlled randomized clinical trials. CMAJ., 182: 1723 - 1729.

9. Li, L. X., Fan, X. N. and Shi, X. M. (2010). Study on the Optimal Stimulating Frequency and Time at "RenZhong” acupoint in “XinNao KaiQiao” Method with the Orthogonal Design. Liaoning Journal of Traditional Chinese Medicine, 37: 1158-1160. In Chinese.

10. Longa, E. Z., Weinstein, P. R., Carlson, S. and Cummins, R. (1989). Reversible middle cerebral artery occlusion without craniectomy in rats. Stroke, 20: 84 - 91.

11. Lou, M., Eschenfelder, C. C., Herdegen, T., Brecht, S. and Deuschl, G. (2004). Therapeutic window for use of hyperbaric oxygenation in focal transient ischemia in rats. Stroke, 35: 578 - 583.

12. Macklin, E. A., Wayne, P. M., Kalish, L. A., Valaskatgis, P., Thompson, J., Pian-Smith, M. C., Zhang, Q., Stevens, S., Goertz, C., Prineas, R. J., Buczynski, B. and Zusman, R. M. (2006). Stop Hypertension with the Acupuncture Research Program (SHARP): results of a randomized, controlled clinical trial. Hypertension, 48: 838 - 845.

13. O'Donnell, M. E., Tran, L., Lam, T. I., Liu, X. B. and Anderson, S. E. (2004). Bumetanide inhibition of the blood-brain barrier Na-K-Cl cotransporter reduces edema formation in the rat middle cerebral artery occlusion model of stroke. $\mathrm{J}$ Cereb Blood Flow Metab., 24: 1046 - 1056.

14. Painovich, J. and Herman, P. M. (2012). Acupuncture in the inpatient acute care setting: a pragmatic, randomized control trial. Evid Based Complement Alternat Med., 2012: 309762.

15. Park, J., White, A. R., James, M. A., Hemsley, A. G., Johnson, P., Chambers, J. and Ernst, E. (2005). Acupuncture for subacute stroke rehabilitation: a Sham-controlled, subject- and assessor-blind, randomized trial. Arch Intern Med., 165: 
2026 - 2031.

16. Rao, P., Zhou, L., Mao, M., Bai, Y., Wen, T. M., Tang, Y. H. and Guo, W. L. (2006). A randomized controlled trial of acupuncture treatment of acute ischemic stroke. Zhongguo Zhen Jiu, 26: 694 - 696. In Chinese.

17. Schaechter, J. D., Connell, B. D., Stason, W. B., Kaptchuk, T. J., Krebs, D. E., Macklin, E. A., Schnyer, R. N., Stein, J., Scarborough, D. M., Parker, S. W., McGibbon, C. A. and Wayne, P. M. (2007). Correlated change in upper limb function and motor cortex activation after verum and sham acupuncture in patients with chronic stroke. J Altern Complement Med., 13: 527 - 532.

18. Si, Q. M., Wu, G. C. and Cao, X. D. (1998). Effects of electroacupuncture on acute cerebral infarction. Acupunct Electrother Res., 23: 117 - 124.

19. Siu, F. K., Lo, S. C. and Leung, M. C. (2005). Electro-acupuncture potentiates the disulphide-reducing activities of thioredoxin system by increasing thioredoxin expression in ischemia-reperfused rat brains. Life Sci., 77: 386 - 399.

20. Swanson, R. A., Morton, M. T., Tsao-Wu, G., Savalos, R. A., Davidson, C. and Sharp, F. R. (1990). A semiautomated method for measuring brain infarct volume. J Cereb Blood Flow Metab., 10: 290 - 293.

21. Sze, F. K., Wong, E., Or, K. K., Lau, J. and Woo, J. (2002). Does acupuncture improve motor recovery after stroke? A meta-analysis of randomized controlled trials. Stroke, 33: 2604 - 2619.

22. Thomas, K. J., MacPherson, H., Thorpe, L., Brazier, J., Fitter, M., Campbell, M. J., Roman, M., Walters, S. J. and Nicholl, J. (2006). Randomised controlled trial of a short course of traditional acupuncture compared with usual care for persistent non-specific low back pain. BMJ., 333: 623.

23. Vickers, A. J., Feinstein, M. B., Deng, G. E. and Cassileth, B. R. (2005). Acupuncture for dyspnea in advanced cancer: a randomized, placebo-controlled pilot trial [ISRCTN89462491]. BMC Palliat Care., 4: 5.

24. Wu, P., Mills, E., Moher, D. and Seely, D. (2010). Acupuncture in poststroke rehabilitation: a systematic review and meta-analysis of randomized trials. Stroke, 41: e171 - e179.

25. Yang, G. Y. and Betz, A. L. (1994). Reperfusion-induced injury to the blood-brain barrier after middle cerebral artery occlusion in rats. Stroke, 25: 1658 - 1664, 1664 - 1665.

26. Yuan, J., Purepong, N., Kerr, D. P., Park, J., Bradbury, I. and McDonough, S. (2008). Effectiveness of acupuncture for low back pain: a systematic review. Spine (Phila Pa 1976)., 33: E887 - E900.

27. Zausinger, S., Hungerhuber, E., Baethmann, A., Reulen, H. and Schmid-Elsaesser, R. (2000). Neurological impairment in rats after transient middle cerebral artery occlusion: a comparative study under various treatment paradigms. Brain Res., 863: 94 - 105.

28. Zhao, D., Liu, J., Wang, W., Zeng, Z., Cheng, J., Liu, J., Sun, J. and Wu, Z. (2008). Epidemiological transition of stroke in China: twenty-one-year observational study from the Sino-MONICA-Beijing Project. Stroke, 39: 1668 - 1674.

29. Zhong, S., Li, Z., Huan, L. and Chen, B. Y. (2009). Neurochemical Mechanism of Electroacupuncture: Anti-injury Effect on Cerebral Function after Focal Cerebral Ischemia in Rats. Evid Based Complement Alternat Med., 6: 51 - 56. 